\title{
Endoscopic Radiofrequency Ablation of the Sacroiliac Joint Complex in the Treatment of Chronic Low Back Pain: A Preliminary Study of Feasibility and Efficacy of a Novel Technique
}

\author{
Won-Suh Choi, ${ }^{1}$ Jin-Sung Kim, ${ }^{2}$ Kyeong-Sik Ryu, ${ }^{2}$ Jung-Woo Hur, ${ }^{2}$ \\ Ji-Hoon Seong, ${ }^{2}$ and Hyun-Jin $\mathrm{Cho}^{2}$ \\ ${ }^{1}$ Department of Neurosurgery, International Naeun Hospital, Gyeonggi, Republic of Korea \\ ${ }^{2}$ Department of Neurosurgery, Seoul St. Mary's Hospital, The Catholic University of Korea, Seoul, Republic of Korea
}

Correspondence should be addressed to Jin-Sung Kim; mddavidk@gmail.com

Received 2 August 2016; Accepted 3 November 2016

Academic Editor: Tsung-Jen Huang

Copyright (C) 2016 Won-Suh Choi et al. This is an open access article distributed under the Creative Commons Attribution License, which permits unrestricted use, distribution, and reproduction in any medium, provided the original work is properly cited.

\begin{abstract}
Background. Radiofrequency ablation (RFA) is a less invasive technique for treatment of sacroiliac joint (SIJ) pain. Objective. To evaluate the feasibility and efficacy of endoscope-guided RFA for the treatment of CLBP from the SIJ complex. Methods. In this retrospective study, the medical records of 17 patients who underwent endoscope-guided RFA of the SIJ complex were reviewed. A bipolar radiofrequency probe was used to lesion the posterior capsule of the SIJ as well as the lateral branches of S1, S2, S3, and the L5 dorsal ramus in multiple locations. We visualized the ablation area using endoscope. We assessed visual analogue scale (VAS) and the Oswestry disability index (ODI) preoperatively, immediately postop, and at 1-, 3-, and 6-month postop outpatient clinic visits. Patient satisfaction of the procedure was assessed in percentages. Results. The mean duration of operation was 20 to 50 minutes. The mean VAS and the ODI scores decreased significantly immediately after the procedure and were kept significantly lower than baseline levels during the follow-up periods. No complications occurred perioperatively and during the follow-up periods. $88.6 \%$ of patients were satisfied with the procedure. Conclusions. Our preliminary results suggest that endoscope-guided RFA may be alternative option to treat CLBP secondary to SIJ complex.
\end{abstract}

\section{Introduction}

Chronic low back pain (CLBP) that lasts for six months or longer is estimated to occur in $60-80 \%$ of the general population in their lifetime [1] and is associated with substantial healthcare costs. The sacroiliac joint (SIJ) complex is one of the major sources of chronic low back pain, accounting for around $10-33 \%$ of the total number of CLBP cases [15]. The SIJ complex consists of the joint capsule, various muscular and ligamentous structures overlying the joint, and neural structures that innervate the SIJ [6]. Current treatment options for SIJ complex-mediated CLBP include intraarticular and periarticular steroid injections, SIJ fusion, and radiofrequency ablation of the neural structures innervating the SIJ. Intra-articular injection of the joint using a mixture of steroids and local anesthetics is a simple procedure and provides quick pain relief, but the effect is short-lived [7]. In addition, SIJ fusion is an invasive surgical procedure that should be reserved for cases refractory to nonoperative measures $[8,9]$. On the other hand, radiofrequency ablation (RFA) of the SIJ complex offers longer-lasting effects and has gained wide attention in the last decade [10], with increasing numbers of reports advocating for its efficacy [7, 11-14]. RFA is usually performed under fluoroscopic guidance. The target structures are the lateral branches of the sacral rami, the dorsal ramus of L5, and the ligamentous structures overlying the joint. However, variations in the pattern of innervation exist between individuals, which provides a challenge for surgeons [15]. Due to these variations, different RFA target locations and techniques have been proposed to overcome 
this difficulty $[16,17]$. Endoscopic radiofrequency ablation has been utilized in the treatment of facetogenic CLBP in a number of clinical reports with favorable results [18-20], but to our knowledge, the efficacy of this technique when applied to SIJ-associated CLBP has not been reported. In this study, we utilized endoscopy for the precise ablation of potential pain generators associated with the SIJ and evaluated the clinical efficacy of this new technique.

\section{Materials and Methods}

The institutional review board at our institution approved this study. The medical records of 17 consecutive patients who underwent RFA of the SIJ for CLBP between April 2011 and December 2015 were reviewed. The inclusion criteria for treatment were as follows: patients with a chief complaint of CLBP with signs and symptoms of SIJ involvement on physical examination and radiological tests such as computed tomography (CT), unresponsive to conservative therapy including oral analgesics and physical therapy, persistent CLBP despite previous lumbosacral operation or pain procedures, and minimum follow-up period of 6 months.

SIJ complex pathology as the main cause of CLBP is difficult to diagnose due to overlapping patterns with other sources of CLBP and varying patterns of pain between individuals $[7,21]$. CT findings of arthropathy or erosion of the SIJ, while not specific, may suggest SIJ based pathology in patients with clinical suspicion [22]. While numerous physical examination methods have been suggested, provocative test was reported to have more reliability in numerous reports $[23,24]$. In order to confirm the SIJ pain as the main source of CLBP, two separate diagnostic intra-articular and multisite lateral sacral branch blocks of the SIJ complex were performed at least 2 weeks apart. If patient experienced 50\% or higher improvement in pain from the baseline according to visual analogue scale (VAS) after each block, SIJ complex was considered to be the main pain generator, and endoscopic RFA was scheduled. Patients with tumors of the SIJ, concern for secondary gain, previous surgery of the SIJ such as SIJ fusion, or other severe comorbid medical conditions were excluded. All patients were followed for a minimum of six months after the procedure in outpatient clinics. Endoscopic RFA of the SIJ complex was performed in the operating room. Patients were discharged the day after the procedure. All patients were followed up at the outpatient clinic at 1, 3, and 6 months after the procedure and annually thereafter.

2.1. Surgical Techniques. All subjects were placed in the prone position on chest rolls on a radiolucent Jackson table. Before beginning the procedure, patients were fully informed of the procedure details. Patients were monitored and maintained communication with the surgeon throughout the procedure.

After sterile prepping and draping, an anteroposterior fluoroscopic view was obtained using a C-arm. A transducer was tilted cephalad approximately 10-15 degrees and was tilted oblique $10-15$ degrees contralaterally to optimally visualize the posterior aspect of the SIJ. The skin entry point was positioned at the inferior aspect of the posterior SIJ, and local anesthetic was injected into the entry point. An 18-gauge needle was docked onto the interosseous ligament overlying the posterior SIJ. Then, a guide wire was advanced through the needle, the needle was removed, and a $0.5 \mathrm{~cm}$ skin incision was made at the entry site. A cannulated obturator was inserted along the guide wire through the skin incision, and a beveled or nonbeveled working cannula of $7.9 \mathrm{~mm}$ diameter was advanced along the obturator until the cannula reached the posterior SIJ. After removing the obturator, the endoscope $(6.9 \mathrm{~mm} \times 5.6 \mathrm{~mm})$ was introduced through the cannula. The final position of the cannula was confirmed with fluoroscopy.

Under endoscopic visualization, the posterior sacroiliac ligament and its overlying soft tissue were ablated using a Trigger-Flex bipolar probe (Elman International, Inc.) that was introduced through the working channel of the endoscope. First, we ablated perforating branches that innervate the posterior capsule of the SIJ. After visual confirmation of the long posterior sacroiliac ligament, we proceeded with RFA along the course of the ligament in the cranial direction to the level of the posterior superior iliac spine (Figure 1). The fluoroscope was then adjusted to obtain an anteroposterior view. Next, using the wanding maneuver of the cannula, the cannula tip was moved along the subcutaneous plane toward the region lateral to the S1-S3 sacral foramina, and a linear multidepth lesion was made along the line connecting the lateral margins of the S1-3 sacral foramina (Figure 2). When uncertain about the position of the RF probe tip, we checked the tip position with the fluoroscope. We attempted to visually confirm the lateral branches exiting the sacral foramina and the branches coursing toward the SIJ when possible to ensure accurate nerve lesioning. Throughout the procedure, we maintained constant communication with the patient to assess the level of pain associated with each stimulus and to identify which stimulus area caused the most pain. Continuous saline irrigation was maintained throughout the procedure to minimize thermal injury to the surrounding structures. After ablation of the target points, the endoscope and cannula were removed. One-point suture with Nylon was used, and sterile dressing was applied.

2.2. Clinical Assessment. Patients were instructed to visit the outpatient clinic at 1, 3, 6, and 12 months after the procedure. Pain intensity and functional disability were assessed via questionnaires with outcome measurements before the procedure, immediately after the procedure, and at each followup outpatient visit. All clinical assessments were performed by a single coresearcher. At each follow-up visit, back and leg pain intensity was assessed using the visual analogue scale (VAS) and the Oswestry disability index (ODI). Additionally, all patients were asked to express their degree of satisfaction with the procedure on a percentage scale.

2.3. Statistical Analysis. Mean VAS scores for back and ODI scores immediately after the procedure, three and six months after, and one year after the procedure were compared to the scores recorded before the procedure. Statistical significance was assessed using paired Student's $t$-tests. $p$ values less than 0.05 were considered to be statistically significant. All statistical analyses were performed using SPSS version 18 (SPSS Inc.). 


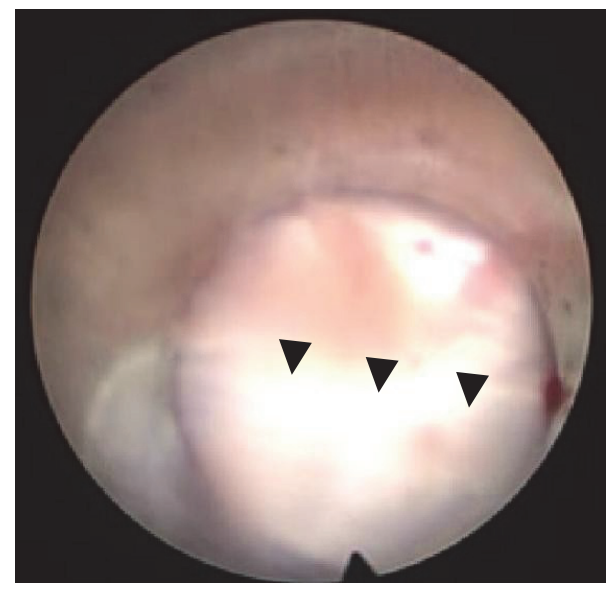

(a)

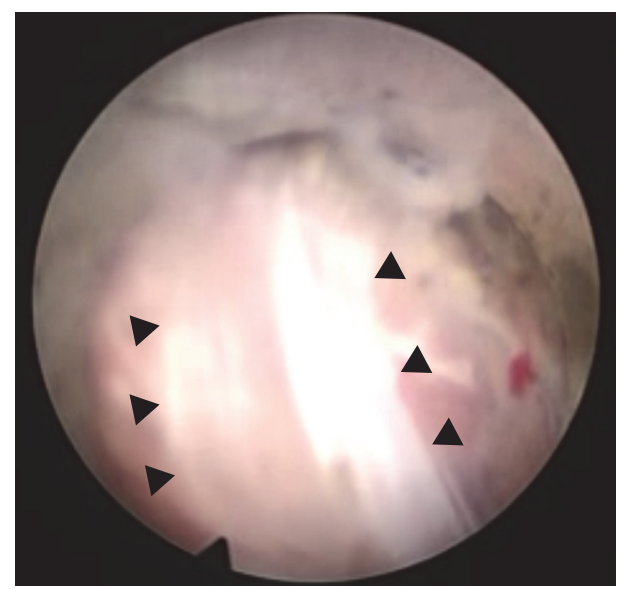

(b)

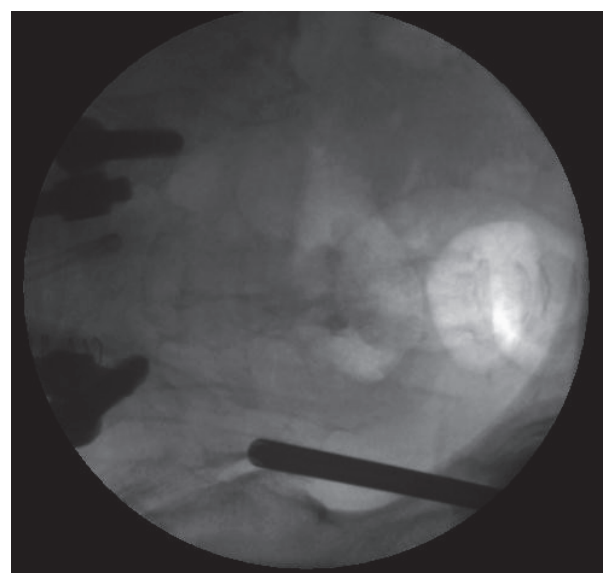

(c)

FIGURE 1: ((a) and (b)) Long posterior ligament (black arrowheads) overlying the posterior capsule of the SIJ. (c) Corresponding position of the cannula tip in the anteroposterior fluoroscopic image.

\section{Results}

In this study, there were 2 male and 15 female patients whose age ranged from 37 to 81 years (mean age $61.9 \pm 11.8$ years). On preoperative lumbosacral MRI, herniated nucleus pulposus was identified in eight patients, spondylolisthesis in four patients, and spinal stenosis in five patients. On CT scan of the pelvis, arthropathy of the SIJ was observed in 11 patients (64.7\%).

Before the RFA procedure, ten patients (58.8\%) had undergone operation or pain procedure on the lumbosacral spine. Four patients (23.5\%) underwent lumbar or lumbosacral interbody fusion and posterior fixation, four $(23.5 \%)$ underwent discectomy or laminectomy, and two (11.8\%) underwent medial branch RFA. Seven patients (41.2\%) underwent nerve block procedures including caudal block, medial branch block, root block, and transforaminal epidural block. All of the ten patients experienced persistent CLBP despite these procedures. Patient demographic data are summarized in Table 1.

RFA was performed on the right side only in eight patients $(47.1 \%)$, on the left side only in five patients $(29.4 \%)$, and on both sides in four patients $(23.5 \%)$. The mean duration of operation from the time of local anesthetic injection to wound closure was $26.6 \pm 22.5(20-50)$ mins per side. All patients were discharged the next day, without perioperative complications such as hematoma collection, wound discharge, or development of acute neurological deficit. The mean VAS scores for back pain decreased from $6.7 \pm 1.41$ preoperatively to $3.6 \pm 1.28,3.2 \pm 1.06,2.8 \pm 1.14$, and $3.1 \pm 1.78$ immediately postoperatively, and at 1,3 , and 6 months' follow-up visits, respectively. All of the follow-up VAS scores were significantly lower than the baseline $(p<$ $0.005)$. The mean ODI score preoperatively was $22.2 \pm 3.36$ and decreased to $14.1 \pm 3.35,13.1 \pm 4.05,12.9 \pm 4.32$, and $12.0 \pm 4.69$ immediately postop and at the 3, 6, and 12-month follow-up visits, respectively. All of the follow-up ODI scores were significantly lower than baseline $(p<0.001)$. Mean patient satisfaction rate was $86.6 \%(70-100)$. These results are summarized in Table 2.

3.1. Example Case. A 69-year-old female presented with chronic low back pain and left buttock pain for the last five years. She had undergone L4-5 and L5-S1 fusion at another 


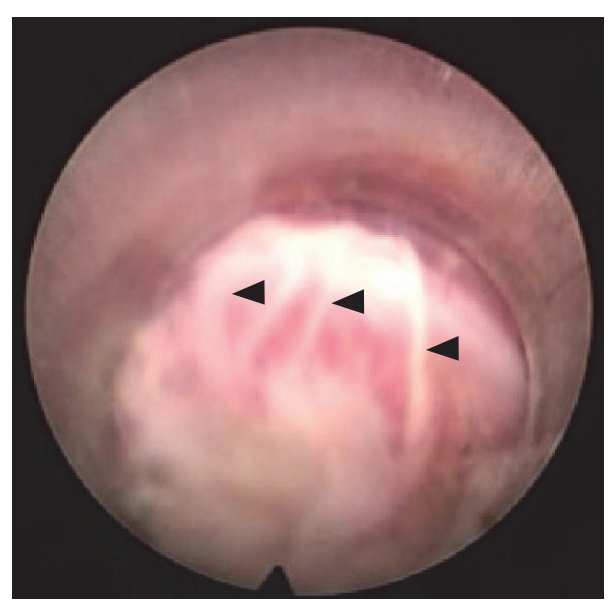

(a)

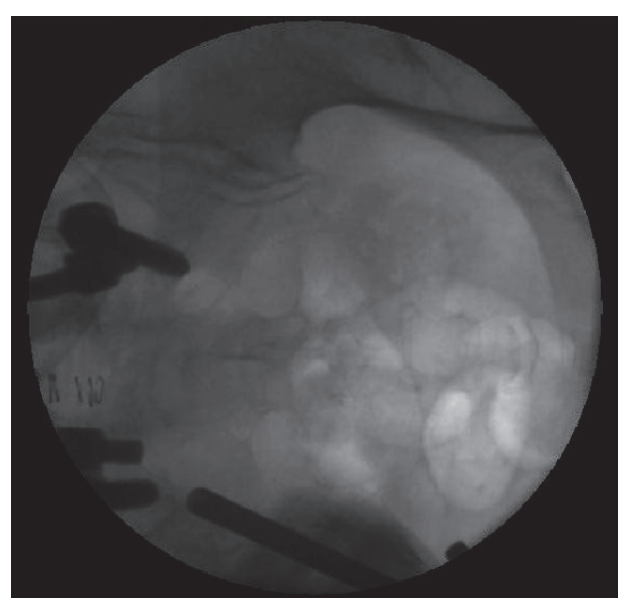

(b)

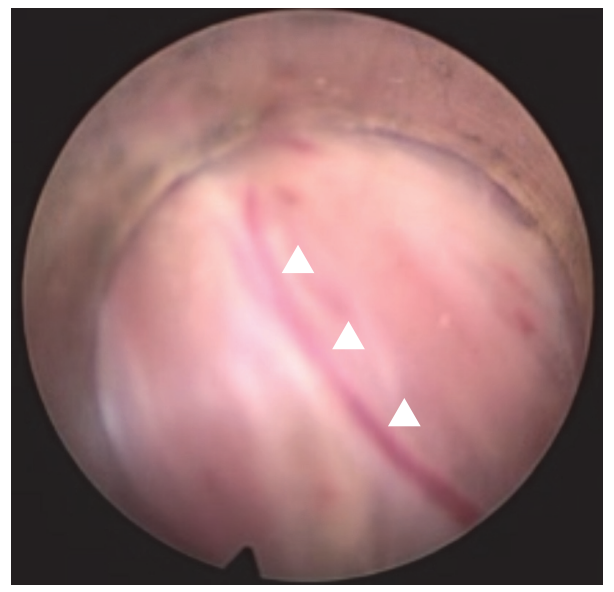

(c)

Figure 2: (a) Lateral sacral branches of S1 as they exit from the S1 foramen (black arrowheads). (b) Corresponding position of the endoscope cannula. (c) Small arteries or veins can often be seen coursing along the nerve branches (white arrowheads), which can help with identification of thin nerve branches.

institution in 2008. She had remained asymptomatic for two years, but in 2010, she started feeling left-sided buttock pain. Conservative treatment with oral analgesics and four separate root blocks and epidural blocks at a local pain clinic were ineffective. CT scan of the pelvis revealed bilateral SIJ arthropathy. Her initial VAS for back pain and her ODI scores were 7 and 40, respectively, and downward pressure on the sacrum with the patient in the prone position elicited severe pain (VAS 8). After two diagnostic SIJ complex injections, her VAS score dropped to 2 points on both occasions. Endoscopeguided RFA was performed one month later.

Local analgesics were applied to the entry point of the skin, which was about $1 \mathrm{~cm}$ above where the buttock pain was elicited. Buttock pain was elicited when the long posterior sacroiliac ligament overlying the posterior articular capsule was stimulated with the RF probe in short bursts (Figures 1(a), 1(b), and $1(\mathrm{c}))$. While maintaining continuous saline irrigation, the painful areas along the length of ligament were coagulated with the RF probe to the level of the posterior superior iliac spine. Next, using the wanding maneuver, the tip of the cannula was gently mobilized in the subcutaneous plane and repositioned next to the lateral margin of the S1 neural foramen. The S1 lateral sacral branches were identified and coagulated in the same manner (Figures 2(a), 2(b), and $2(c))$. Ablation of a targeted area was stopped when no further pain was elicited upon additional stimulation. The S2 and S3 lateral branches were ablated in a similar manner (Figure 3).

\section{Discussion}

The SIJ complex is increasingly being recognized as a major source of CLBP, and, yet, the exact pain generating mechanisms and anatomical properties of the SIJ complex have not been fully established. The literature surrounding the treatment of SIJ complex-mediated pain is still quite sparse. Diagnosing SIJ complex pain remains largely a diagnosis of exclusion. In SIJ complex pain, patients tend to complain of buttock pain, but many experience lower leg pain on the involved side as well, which can be confused with radiculopathy or referred pain from other low back structures 
TABle 1: Patient demographic data.

\begin{tabular}{|c|c|c|c|c|c|c|}
\hline $\begin{array}{l}\text { Patient } \\
\text { number }\end{array}$ & Age & Gender & $\begin{array}{c}\text { Follow-up } \\
\text { period (months) }\end{array}$ & $\begin{array}{c}\text { Side of } \\
\text { procedure }\end{array}$ & Other diagnoses & Previous operations/procedures \\
\hline 1 & 56 & $\mathrm{~F}$ & 49 & Right & HNP, L4-5, Lt. & Root block, L4, 5, Lt. \\
\hline 2 & 70 & $\mathrm{~F}$ & 37 & Left & $\begin{array}{c}\text { Extraforaminal HNP, L5-S1, } \\
\text { Lt. }\end{array}$ & Transforaminal epidural block, L5-S1, Lt. \\
\hline 3 & 74 & $\mathrm{~F}$ & 36 & Both & HNP, L3-4, 4-5, 5-S1 & $\begin{array}{l}\text { Caudal block } \\
\text { medial branch block, L3-4, 4-5, 5-S1, both }\end{array}$ \\
\hline 4 & 73 & $\mathrm{~F}$ & 35 & Both & Spinal stenosis, L3-4, 4-5 & Medial branch block, L3-4, 4-5, both \\
\hline 5 & 76 & $\mathrm{~F}$ & 35 & Left & HNP, L4-5, Rt. & $\begin{array}{l}\text { (1) Decompressive hemilaminectomy, L4, Rt. } \\
\text { (2005) } \\
\text { (2) Medial branch RFA, L4, 5, Rt. (2006) }\end{array}$ \\
\hline 6 & 81 & $\mathrm{~F}$ & 22 & Right & Spinal stenosis, L3-4, 4-5 & PLIF, L3-4, 4-5 (2008) \\
\hline 7 & 37 & $\mathrm{~F}$ & 22 & Right & Spinal stenosis, L4-5, L5-S1 & Root block, L4, 5, Rt. \\
\hline 8 & 72 & $\mathrm{~F}$ & 21 & Left & Spinal stenosis, L5-S1 & PLIF, L5-S1 (2001) \\
\hline 9 & 61 & $\mathrm{~F}$ & 15 & Left & Spondylolisthesis, L4-5 & $\begin{array}{l}\text { 2014.10.8 MIS TLIF } 45 \\
\text { (post-procedure) }\end{array}$ \\
\hline 10 & 57 & $\mathrm{~F}$ & 13 & Right & Spinal stenosis, L4-5 & PLIF, L4-5 (2012) \\
\hline 11 & 48 & $\mathrm{~F}$ & 13 & Right & HNP, L3-4, Rt. & Transforaminal epidural block, L3-4, Rt. \\
\hline 12 & 58 & $\mathrm{~F}$ & 12 & Right & Spondylolisthesis, L3-4 & $\begin{array}{l}\text { Caudal block, epidural block, L3-4 } \\
\text { Trigger point injection of paravertebral muscles }\end{array}$ \\
\hline 13 & 56 & $\mathrm{~F}$ & 12 & Left & HNP, L5-S1, Rt. & Discectomy, L5-S1, Rt. (2013) \\
\hline 14 & 58 & $\mathrm{~F}$ & 10 & Both & Facet arthropathy, L4-5, Lt. & $\begin{array}{l}\text { Medial branch RFA, } \\
\text { L4, 5, Lt. (2013) }\end{array}$ \\
\hline 15 & 60 & $\mathrm{~F}$ & 9 & Both & Spondylolisthesis, L4-5 & PLIF, L4-5 (2013) \\
\hline 16 & 47 & $\mathrm{M}$ & 9 & Right & HNP, L5-S1, Lt. & Discectomy, L5-S1, Lt. (2013) \\
\hline 17 & 69 & $\mathrm{~F}$ & 8 & Right & $\begin{array}{l}\text { Spondylolisthesis, L4-5, } \\
\text { L5-S1 }\end{array}$ & PLIF, L4-5, 5-S1 (2008) \\
\hline
\end{tabular}

TABLE 2: Preoperative and postoperative clinical data.

\begin{tabular}{lccccc}
\hline & $\begin{array}{c}\text { Mean preoperative } \\
\text { scores }\end{array}$ & $\begin{array}{c}\text { Mean immediate } \\
\text { postoperative scores }\end{array}$ & $\begin{array}{c}\text { Mean 1-month follow-up } \\
\text { scores }\end{array}$ & $\begin{array}{c}\text { Mean 3-month } \\
\text { follow-up scores }\end{array}$ & $\begin{array}{c}\text { Mean 6-month } \\
\text { follow-up scores }\end{array}$ \\
\hline VAS & $6.7 \pm 1.41$ & $3.6 \pm 1.28$ & $3.2 \pm 1.06$ & $2.8 \pm 1.14$ & $3.1 \pm 1.78$ \\
ODI & $22.2 \pm 3.36$ & $14.1 \pm 3.35$ & $13.1 \pm 4.05$ & $12.9 \pm 4.32$ & $12.0 \pm 4.69$ \\
\hline
\end{tabular}

[25]. There is no single reliable test that can identify the SIJ complex as the main pain generator in CLBP, and, in the majority of cases, there are a number of possible pain generators that are equally likely to contribute to the CLBP. For these reasons, accurate diagnosis and successful treatment of SIJrelated pain provide a challenge for surgeons, often causing delay in recognition of SIJ complex as the main source of CLBP. Recognizing the SIJ as the main pain generator takes time and effort, since SIJ complex pain is often only diagnosed after all other modalities fail to treat the patient's CLBP. In our case series, all 17 patients had competing pathologies of the lumbar spine, including herniated lumbar disks, spinal stenosis, spondylolisthesis, and facet joint arthropathy that may have contributed to their symptoms. All had received one or more pain procedures at the lumbar level, including root blocks, epidural blocks, epidural neurolysis, facet joint blocks, and medial branch blocks/neurotomy with minimal or unsatisfactory results. Of the 17 patients, 7 (41.1\%) had previously undergone some form of surgical procedure at the lumbar level, including five cases of interbody fusion and two cases of discectomy, which did not satisfactorily alleviate their CLBP. The average duration of symptoms prior to the diagnosis of SIJ complex pain was $2.8 \pm 6.5$ years (6 months10 years).

The SIJ complex consists of an articular region, a posterior ligamentous region, and a dorsal ligamentous region, which support the joint [26]. Neural innervations are found in both the posterior capsule of the joint and in the posterior sacroiliac and interosseous ligaments [6]. Although many studies have used intra-articular injections both to provide relief and to identify the origin of the SIJ pain, results from recent studies indicate that periarticular ligamentous structures may contribute more to SIJ pain than the articular region itself [27, 28 ], suggesting that the periarticular ligamentous structures are better targets for treatment. Dreyfuss et al. demonstrated that multisite, multidepth lateral sacral branch blocks are 

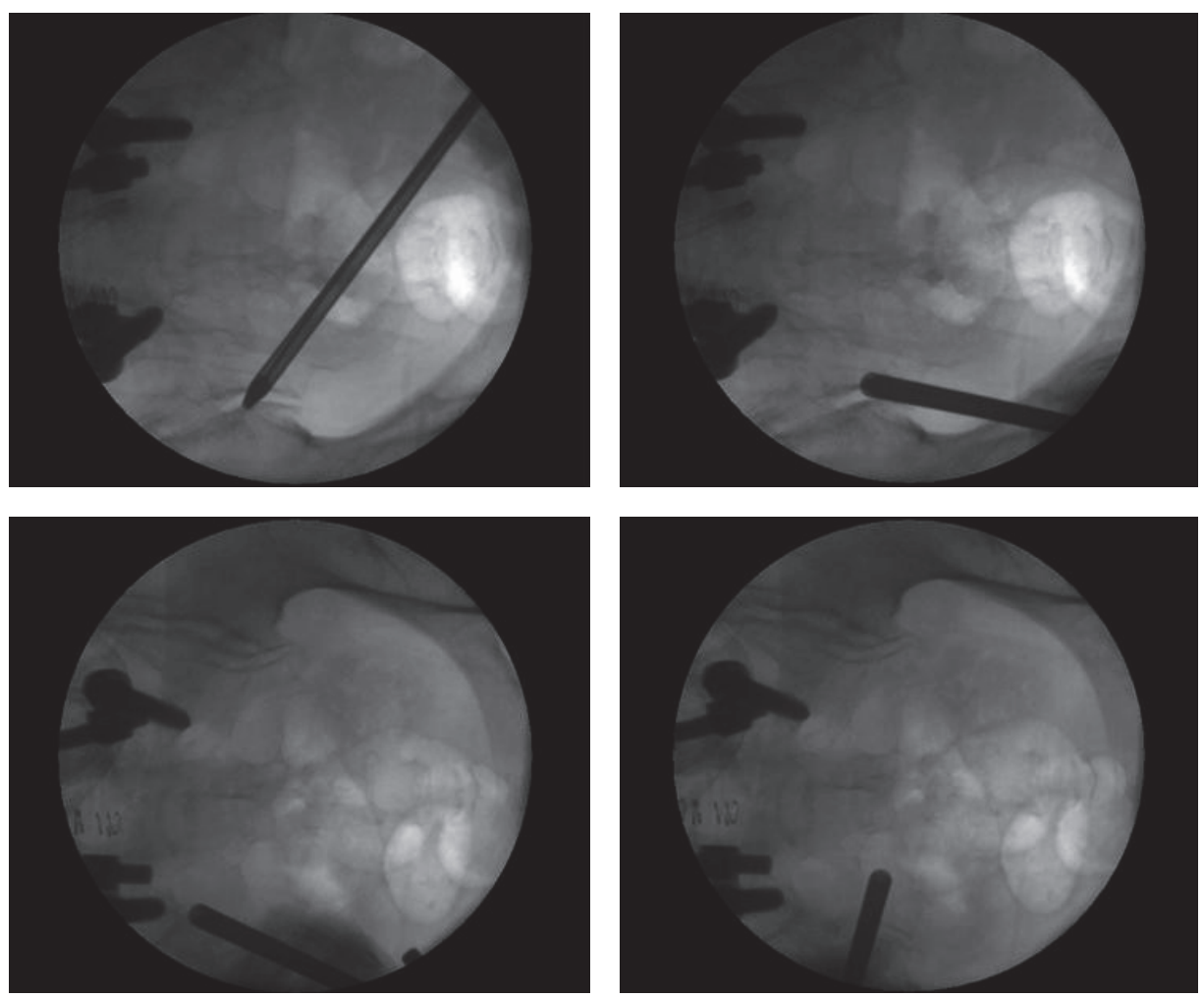

FIgURE 3: Fluoroscopic view of the endoscopic cannula tip in various positions during the procedure. The cannula tip can be moved in the subcutaneous plane and can be repositioned without causing much discomfort. If patients did experience discomfort, an additional lidocaine injection was applied.

more reliable than intra-articular injections for identifying the SIJ complex as the main pain generator, and a recent systemic review by King et al. also supports this view. When performing diagnostic blocks, we targeted the intra-articular joint itself as well as the lateral sacral branches of S1-3 and the L5 dorsal ramus, since these will be the targets for RFA. Additionally, compared to intra-articular injection alone, relief after injection of these structures would be a better predictor of favorable outcomes after RFA.

The lateral branches of L5-S4, especially the branches of S1-S3 innervating the sacroiliac joint, are quite variable between individual patients in their course, branches, and location. To ablate all pain generators, a large lesion area would be required $[16,17]$. Current techniques to accomplish these lesions include a periforaminal approach and a lateral sacral crest approach. The periforaminal approach involves making a series of lesions around the lateral border of the S1S3 sacral foramina using a monopolar or bipolar RF probe. However, as described by Roberts et al. [17], it is often difficult to achieve a clear view of the foramina under fluoroscopy. The lateral sacral technique involves creating a lesion strip along the lateral sacral crest at regular intervals, effectively ablating the fine plexus of lateral branches overlying this region. This technique also has disadvantages in that the lateral crest is not easy to identify under fluoroscopy alone.

For both the periforaminal approach and the lateral sacral crest approach, multiple skin punctures are made in order to place the RF probe, which can result in patient discomfort. Because the cannula for the endoscope is considerably thicker and more rigid than the RF probe itself, it was possible to reach the periforaminal regions of the S1-3 sacral foramina through a single incision using the endoscope wanding maneuver. Notably, the wanding maneuver did not cause the patients more discomfort. If the procedure was uncomfortable, we offered the patient light sedation with midazolam. With the aid of the endoscope, we achieved better visualization of bony landmarks as well as the lateral branches of S1-S3 when possible. The branches of the posterior rami of S1-S3 travel deep to the long posterior sacroiliac and sacrotuberous ligaments. According to a cadaveric study by Roberts et al. [17], the diameter of the S1-S3 branches ranges from 0.21 to $1.51 \mathrm{~mm}$, and in many cases, the lateral branches could not be identified with certainty. If gentle stimulation of the suspected lateral branch with the RF probe elicited pain, we ablated the branch. Except for one patient, all procedures were performed with the patient under local anesthesia or light sedation, and communication with the patient was maintained throughout the procedure.

Another advantage of direct visualization with the endoscope is that it affords us the ability to identify areas that have already been ablated. This allows us to avoid damaging the soft tissue with excessive lesioning of the same region. Avoiding repeated lesioning helped curb complications such as postprocedural pain and dysesthesia (Figure 4). 


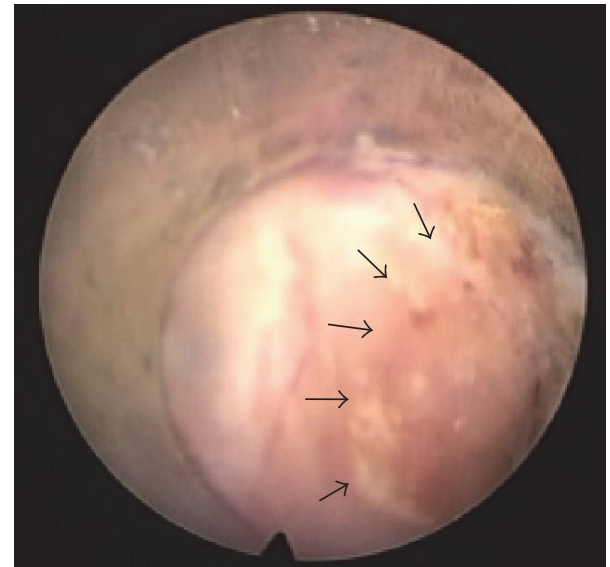

Figure 4: Under endoscopic view, it is possible to clearly discern areas that have already been ablated (surrounded by arrows) and which areas have not. It is also possible to gauge the depth of the ablation.

Our study has several limitations. First, this is a retrospective study with a limited number of cases. Second, no direct comparison was made with conventional fluoroscopicguided RFA method in the clinical results and perioperative parameters. Third, patients were not categorized by their VAS and ODI scores before the procedure or previous operation or procedure, and clinical effects of endoscopic RFA procedure could be confounded by other variables. We plan to conduct a randomized clinical trial comparing conventional RFA and endoscopic RFA in the future.

\section{Conclusions}

Our preliminary results suggest that endoscope-guided RFA may be alternative option to treat CLBP secondary to SIJ complex pain with favorable clinical outcomes, including a long-term pain-free period and improved physical function with minimal complications.

\section{Competing Interests}

The authors declare that there is no conflict of interests regarding the publication of this paper.

\section{References}

[1] M. V. Boswell, A. M. Trescot, S. Datta et al., "Interventional techniques: evidence-based practice guidelines in the management of chronic spinal pain," Pain Physician, vol. 10, no. 1, pp. 7-111, 2007.

[2] A. C. Schwarzer, C. N. Aprill, and N. Bogduk, "The sacroiliac joint in chronic low back pain,” Spine, vol. 20, no. 1, pp. 31-37, 1995.

[3] F. D'Orazio, L. M. Gregori, and M. Gallucci, "Spine epidural and sacroiliac joints injections-when and how to perform," European Journal of Radiology, vol. 84, no. 5, pp. 777-782, 2015.

[4] J.-Y. Maigne, A. Aivaliklis, and F. Pfefer, "Results of sacroiliac joint double block and value of sacroiliac pain provocation tests in 54 patients with low back pain," Spine, vol. 21, no. 16, pp. 1889$1892,1996$.
[5] J. N. Sembrano and D. W. Polly, "How often is low back pain not coming from the back?” Spine, vol. 34, no. 1, pp. E27-E32, 2009.

[6] V. Bowen and J. D. Cassidy, "Macroscopic and microscopic anatomy of the sacroiliac joint from embryonic life until the eighth decade," Spine, vol. 6, no. 6, pp. 620-628, 1981.

[7] S. P. Cohen, Y. Chen, and N. J. Neufeld, "Sacroiliac joint pain: a comprehensive review of epidemiology, diagnosis and treatment," Expert Review of Neurotherapeutics, vol. 13, no. 1, pp. 99-116, 2013.

[8] J. M. Buchowski, K. M. Kebaish, V. Sinkov, D. B. Cohen, A. N. Sieber, and J. P. Kostuik, "Functional and radiographic outcome of sacroiliac arthrodesis for the disorders of the sacroiliac joint," Spine Journal, vol. 5, no. 5, pp. 520-529, 2005.

[9] K. A. Giannikas, A. M. Khan, M. T. Karski, and H. A. Maxwell, "Sacroiliac joint fusion for chronic pain: a simple technique avoiding the use of metalwork," European Spine Journal, vol. 13, no. 3, pp. 253-256, 2004.

[10] R. C. Cox and J. D. Fortin, "The anatomy of the lateral branches of the sacral dorsal rami: implications for radiofrequency ablation," Pain Physician, vol. 17, no. 5, pp. 459-464, 2014.

[11] W. Stelzer, M. Aiglesberger, D. Stelzer, and V. Stelzer, "Use of cooled radiofrequency lateral branch neurotomy for the treatment of sacroiliac joint-mediated low back pain: a large case series," Pain Medicine (United States), vol. 14, no. 1, pp. 2935, 2013.

[12] P. Vanelderen, K. Szadek, S. P. Cohen et al., "13. Sacroiliac joint pain," Pain Practice, vol. 10, no. 5, pp. 470-478, 2010.

[13] S. M. Aydin, C. G. Gharibo, M. Mehnert, and T. P. Stitik, "The role of radiofrequency ablation for sacroiliac joint pain: a metaanalysis," $P M$ \& $R$, vol. 2, no. 9, pp. 842-851, 2010.

[14] F. M. Ferrante, L. F. King, E. A. Roche et al., "Radiofrequency sacroiliac joint denervation for sacroiliac syndrome," Regional Anesthesia and Pain Medicine, vol. 26, no. 2, pp. 137-142, 2001.

[15] S. P. Cohen and S. Abdi, "Lateral branch blocks as a treatment for sacroiliac joint pain: A Pilot Study," Regional Anesthesia and Pain Medicine, vol. 28, no. 2, pp. 113-119, 2003.

[16] K. Y. Ho, M. A. Hadi, K. Pasutharnchat, and K. H. Tan, "Cooled radiofrequency denervation for treatment of sacroiliac joint pain: two-year results from 20 cases," Journal of Pain Research, vol. 6, pp. 505-511, 2013.

[17] S. Roberts, R. Burnham, K. Ravichandiran, A. Agur, and E. Loh, "Cadaveric study of sacroiliac joint innervation: implications for diagnostic blocks and radiofrequency ablation," Regional Anesthesia and Pain Medicine, vol. 39, no. 6, pp. 456-464, 2014.

[18] Z.-Z. Li, S.-X. Hou, W.-L. Shang, K.-R. Song, and W.-W. Wu, "Evaluation of endoscopic dorsal ramus rhizotomy in managing facetogenic chronic low back pain," Clinical Neurology and Neurosurgery, vol. 126, pp. 11-17, 2014.

[19] S. Y. Jeong, J. S. Kim, W. S. Choi, J. W. Hur, and K. S. Ryu, "The effectiveness of endoscopic radiofrequency denervation of medial branch for treatment of chronic low back pain," Journal of Korean Neurosurgical Society, vol. 56, no. 4, pp. 338-343, 2014.

[20] A. Yeung and S. Gore, "Endoscopically guided foraminal and dorsal rhizotomy for chronic axial back pain based on cadaver and endoscopically visualized anatomic study," International Journal of Spine Surgery, vol. 8, pp. 23-23, 2014.

[21] N. C. Nacey, J. T. Patrie, and M. G. Fox, "Fluoroscopically Guided Sacroiliac Joint Injections: comparison of the Effects of Intraarticular and Periarticular Injections on Immediate and Short-Term Pain Relief," American Journal of Roentgenology, vol. 207, no. 5, pp. 1055-1061, 2016. 
[22] H. Elgafy, H. B. Semaan, N. A. Ebraheim, and R. J. Coombs, "Computed tomography findings in patients with sacroiliac pain," Clinical Orthopaedics and Related Research, no. 382, pp. 112-118, 2001.

[23] M. Laslett and M. Williams, "The reliability of selected pain provocation tests for sacroiliac joint pathology," Spine, vol. 19, no. 11, pp. 1243-1249, 1994.

[24] P. Van Der Wurff, R. H. M. Hagmeijer, and W. Meyne, "Clinical tests of the sacroiliac joint. A systematic methodological review. Part 1: reliability," Manual Therapy, vol. 5, no. 1, pp. 30-36, 2000.

[25] J. D. Fortin, A. P. Dwyer, S. West, and J. Pier, "Sacroiliac joint: pain referral maps upon applying a new injection/arthrography technique part i: asymptomatic volunteers," Spine, vol. 19, no. 13, pp. 1475-1482, 1994.

[26] L. Kapural, F. Nageeb, M. Kapural, J. P. Cata, S. Narouze, and N. Mekhail, "Cooled radiofrequency system for the treatment of chronic pain from sacroiliitis: the first case-series," Pain Practice, vol. 8, no. 5, pp. 348-354, 2008.

[27] A. Vleeming, H. B. Albert, H. C. Östgaard, B. Sturesson, and B. Stuge, "European guidelines for the diagnosis and treatment of pelvic girdle pain," European Spine Journal, vol. 17, no. 6, pp. 794-819, 2008.

[28] E. Murakami, Y. Tanaka, T. Aizawa, M. Ishizuka, and S. Kokubun, "Effect of periarticular and intraarticular lidocaine injections for sacroiliac joint pain: prospective comparative study," Journal of Orthopaedic Science, vol. 12, no. 3, pp. 274-280, 2007. 


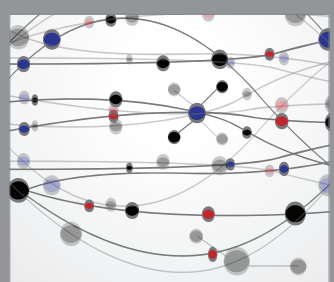

The Scientific World Journal
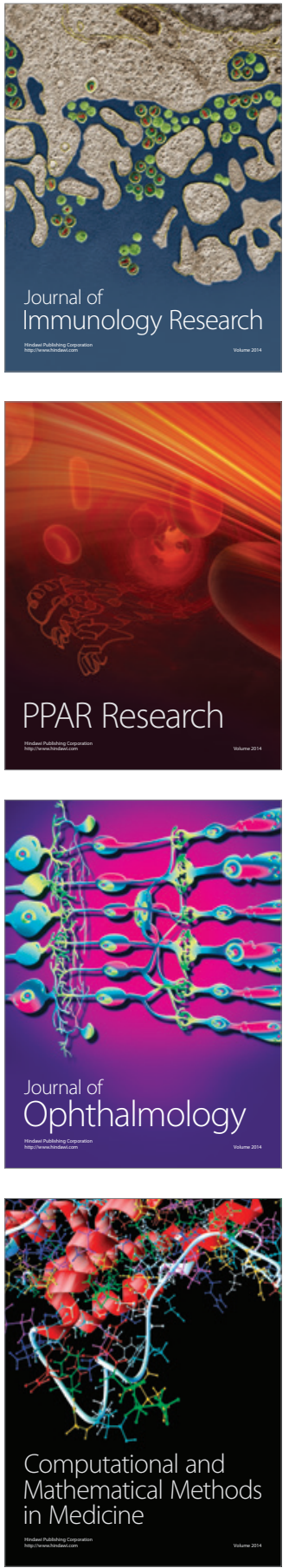

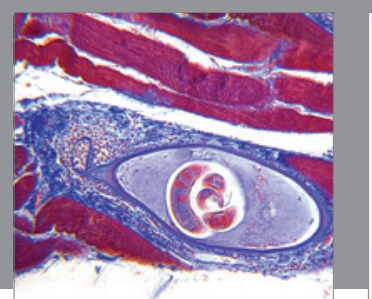

Gastroenterology Research and Practice

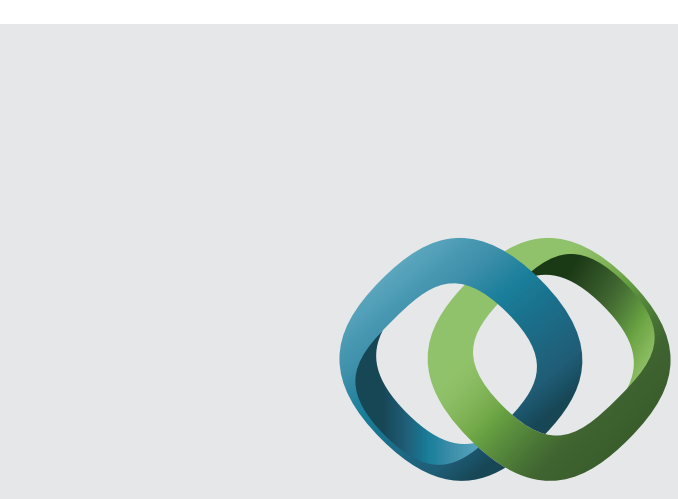

\section{Hindawi}

Submit your manuscripts at

http://www.hindawi.com
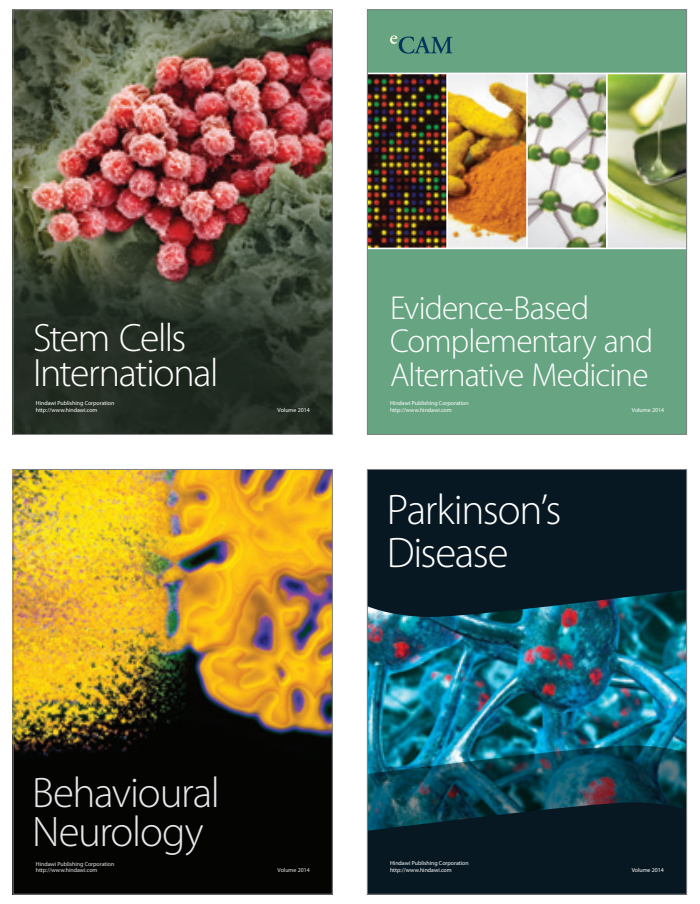
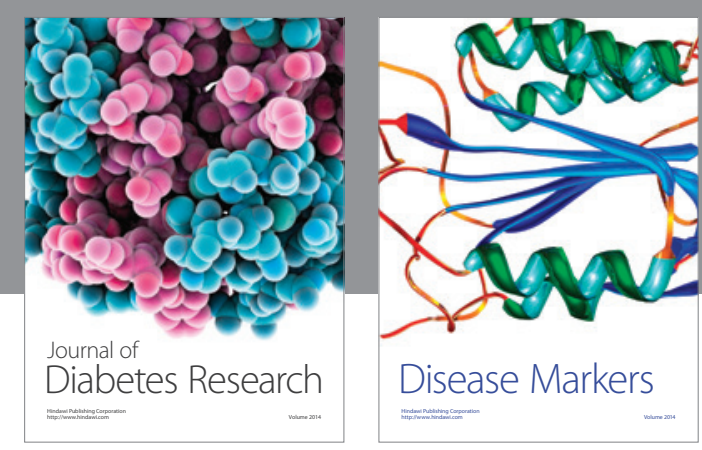

Disease Markers
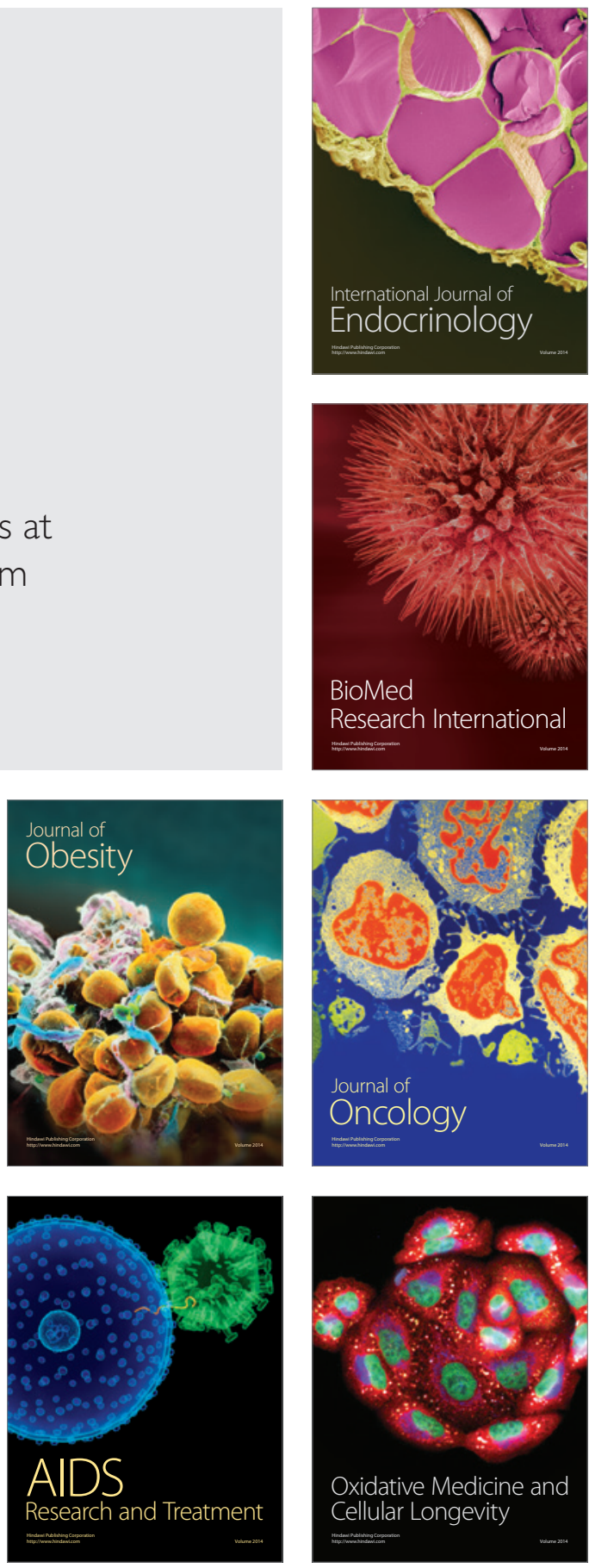